\title{
Structure, dynamics and function of the monooxygenase P450 BM-3: insights from computer simulations studies
}

\author{
Danilo Roccatano \\ School of Engineering and Science, Jacobs University Bremen, Campus Ring 1, \\ 28759 Bremen, Germany. \\ Email: Danilo.Roccatano@gmail.com
}

\begin{abstract}
The monooxygenase P450 BM-3 is a NADPH-dependent fatty acid hydroxylase enzyme isolated from soil bacterium Bacillus megaterium. As a pivotal member of cytochrome P450 superfamily, it has been intensely studied for the comprehension of structure-dynamics-function relationships in this class of enzymes. In addition, due to its peculiar properties, it is also a promising enzyme for biochemical and biomedical applications. However, despite the efforts, the full understanding of the enzyme structure and dynamics is not yet achieved. Computational studies, in particular molecular dynamics (MD) simulations, are given important contributes to this endeavor by providing new insights at atomic level on the correlations between structure, dynamics and function of the protein. This topical review will summarize computational studies based on MD simulations of the cytochrome P450 BM-3 and it will give an outlook on future directions.
\end{abstract}




\section{Introduction}

The cytochrome P450 monooxygenase is a large superfamily of enzymes present in most of the living organisms and comprises numerous enzymes characterized by a unique fold. These proteins are located either in the membrane, or in the cytosol of the cells. The name P450 is after the characteristic absorption maximum of the Soret band observed in their optical spectrum. Upon ligation with carbon monoxide, the maximum shifts from 420 to 450 $\mathrm{nm}$ giving a characteristic red color (Estabrook, 2003) to the enzymes. This absorption band is produced by the iron ion of the Fe-protoporphyrin IX (heme group) present in the catalytic domain of the protein. The function of these enzymes is mainly the oxidation of organic substrates to alcohols, epoxides or aldehydes to make them more soluble, and facilitate their transport, metabolism and/or catabolism. For example, the different isoforms of a human cytochrome P450 present in our liver are responsible for the depuration of our body from toxic xenobiotic substances accidently ingested with our meals, or taken as medicines. In order to perform enzymatic reactions, P450's need a source of electrons, provided by different types of electron transport proteins (reductases), which are used to activate the iron in the heme domain. P450 monooxygenases oxidize a broad range of substrates including fatty acids, alcohols, aliphatic and aromatic hydrocarbons, which are often poorly soluble in water and have $\mathrm{K}_{\mathrm{m}}$ values in the $\mathrm{mM}$ range.

The cytochrome P450 BM-3 (CYP102A1) is an important representative of this class of enzymes (Chefson and Auclair, 2006; Guengerich, 2001; Wong, 1998). It was isolated from soil bacterium Bacillus megaterium (Narhi and Fulco, 1986, 1987a)) in which it probably plays a role in detoxification of polyunsaturated fatty acids. It is a self-sufficient enzyme containing both the heme and a diflavin reductase domain, and it takes electrons directly from the nicotinamide adenine dinucleotide phosphate (NADPH), the primary electron source of the cells. For this property it is classified in the Class VIII of cytochrome P450 superfamily. This peculiar fusion architecture confers to the enzyme an efficient electron transfer (ET) rate, and a high catalytic turnover (it can oxidase 285 molecules of arachidonic acid each second)(Munro et al., 2002a). In addition, it can be easily expressed as recombinant protein in E.coli bacteria (Munro et al., 1994; Warman et al., 2005) that make easier its production and handling with respect to other monooxygenases of the same family (Munro et al., 2007). For this reason, the P450 BM-3 is also an attractive enzyme for biochemical and biotechnological applications (Di Nardo and Gilardi, 2012; Whitehouse et al., 2012). 
As a pivotal member of the P450 superfamily, it has been extensively studied as model system for the comprehension of structure-dynamics-function relationships of the P450 enzymes. Indeed, the abundance of structural, biochemical and mutagenesis data makes it one of the most studied enzymes (Girvan et al., 2006; Munro et al., 2002a). A comprehensive review of these studies has been recently published (Whitehouse et al., 2012), and the reader can refer to it for more comprehensive information and references.

The dynamics of the enzyme and its role in the enzymatic activity is still puzzling since the structure of the complete protein is not yet clarified. Sedimentation and size exclusion chromatography measurements give evidences that the enzyme form in solution a dimer (Black and Martin, 1994) but it is not yet clear how the two monomeric units are assembled together (Girvan et al., 2011). Although the biological assembly structure of the enzyme is not yet disclosed, the available crystallographic structures of the three domains offer a trove of useful information to answer many important questions on the dynamics of the enzyme and the correlation with its function. These questions concern different molecular aspects of the P450 BM-3 dynamics. For example, how substrates diffuse in and out the active site? In which manner the protein collective motions concertize its function and eventually control the electron transfer between the heme domain and the reductase domains? A powerful approach to answers these questions is the study of the enzyme using molecular modeling. In particular, molecular dynamics (MD) simulation method is one of the most powerful approach to analyse protein dynamics at different scale of time and space (Berendsen, 2007). Nowadays, it is broadly used to complement experimental data in devising models of structure and dynamics of molecular systems. The quality and accuracy of the results from MD simulations is mirroring the continuous increase of computer performances. Indeed, in the last few years, the availability of low-cost supercomputer power, stirred by videogames consumers, is accomplishing the amazing prospective of using the MD simulations as a "microscope" for molecular biology investigations (Dror et al., 2012; Lee et al., 2009).

Many MD simulation studies have been performed on P450 BM-3 to understand its molecular properties and functions. In this topical review, the main results of these researches are summarized with an outline on the future directions.

\section{Structural organization of the P450 BM-3}

The cytochrome P450 BM-3 comprises a total of 1048 amino acids organized in three structural domains: the heme domain (HEME, amino acids 1-470), the flavin mononucleotide domain (FMN, 471-664), and the flavin adenine dinucleotide (FAD, 665-1048). The last two 
domains form the diflavine reductase part of the enzyme. The active site of the enzyme, containing the $\mathrm{Fe}(\mathrm{III})$-protoporphyrin IX cofactor, is located in the HEME domain (Whitehouse et al., 2012).

As aforementioned, the structure of the whole protein is not yet available since so far its high flexibility and poor stability of domains linker regions vanished all the attempts to crystallize it. In addition, experimental evidences indicate that the functional form of the enzyme in solution is a dimer.

Fortunately, several crystal structures of the isolated structural domains of the protein have been resolved (see Figure 1). A search in the in the Protein Data Bank (PDB, www.pdb.org) on December 2014 gives 68 x-ray crystallographic structures of P450 BM-3 domains. So far, this collection of structures is the largest available for a cytochrome P450 enzyme. The first crystallographic structure of the HEME domain was determined in 1993 (Ravichandran et al., 1993), and it was the second structure of a cytochrome P450 enzyme to be ever solved. Currently 65 crystallographic structures of the HEME domain are deposited in PDB as wild-type (WT) protein and mutants. Only one structure of the FMN domain, in complex with the HEME domain (PDB-code: 1BYV), is present in the PDB (Sevrioukova et al., 1999). Finally, the last piece of this jiggle saw puzzle protein, the structure of the FAD domain, was recently solved (Joyce et al., 2012).

The enzyme catalyzes the reaction using as electron source NADPH molecules that supply electrons to the heme iron via the two diflavin reductase domains. The FAD domain receives the two electrons from the NADPH and transfers them, one at the time, to the FMN domain that is the intermediate electron donor to the heme iron (Sevrioukova and Peterson, 1996; Sevrioukova et al., 1996b). The HEME domain accepts electrons from the FMN domain only when the substrate is in the active site. This control on the electron transfer is operated by a conformational change of the HEME domain induced by the substrate binding that displaces the water coordinating iron changing its redox potential (Munro et al., 2002a; Munro et al., 1999; Whitehouse et al., 2012). The isolated HEME domain can also fully preserve its catalytic functionality once a source of electrons is provided. Several experimental studies have proved that its catalytic activity can be driven by direct (electrodes) or indirect (electron mediators molecules) electron sources (Nazor et al., 2008; Nazor and Schwaneberg, 2006; Udit et al., 2004). Finally, many mutants of this domain with improved enzymatic activity and/or changed specificity have been obtained using protein engineering and the reader can refer to (Whitehouse et al., 2012) for a recent detailed list of them.

The catalytic mechanism of the P450 BM-3 is very similar to the one of other P450 monooxygenases, and it has been keenly investigated both experimentally and theoretically (Meunier et al., 2004; Sligar et al., 2005). However, there are many puzzling molecular 
aspects (mainly related to the dynamics of the enzyme) that still remains unrevealed. The properties of the enzyme that so far have been studied using MD simulations and published in literature are the followings:

1) Accessibility of the substrate in the access channel.

2) Rational design of the active site.

3) Conformational changes of the enzyme upon substrate binding.

4) Effect of non-natural environments on the enzymatic activity.

5) Coupling of the ET with the protein dynamics.

A
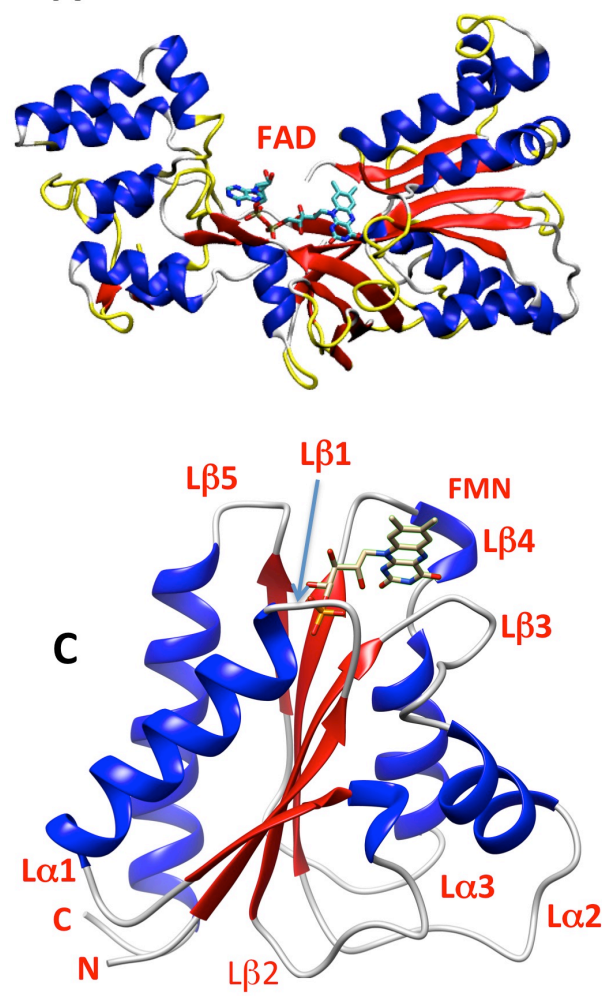
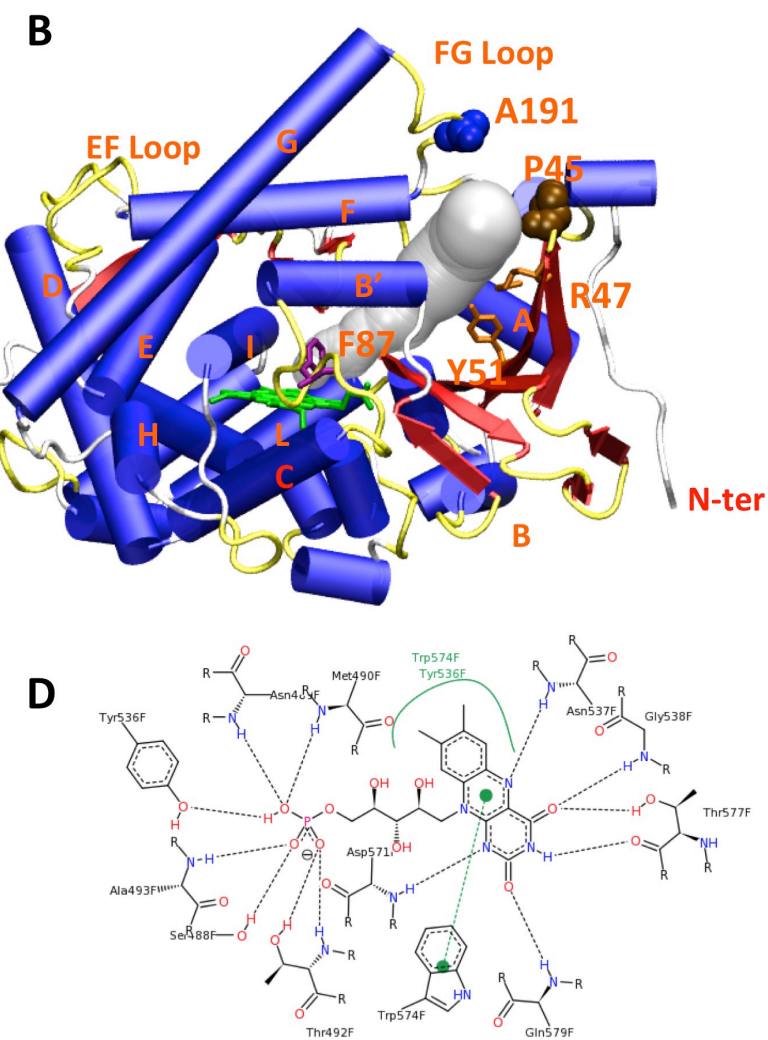

Figure 1: Crystal structures of the three structural domains of P450 BM-3. The secondary structure of the molecule is represented in ribbon and cartoon style and only relevant secondary structure elements are labelled. The important amino acids and cofactors are shown in stick representation and labeled. The protein images were prepared using the program VMD (Humphrey et al., 1996). A: FAD domain (pdb-code: 4DQK ). B: HEME domain (PDB-code: 1BU7, unit B). The heme cofactor is colored in green. The silver tube in the protein represents the volume of the substrate channel as calculated by the program CAVER (Petřek et al., 2006). C: FMN domain (PDB-code: 1BVY). D) FMN cofactor and its interaction with the amino acids in the protein generated by the program PosView (Stierand et al., 2006). 


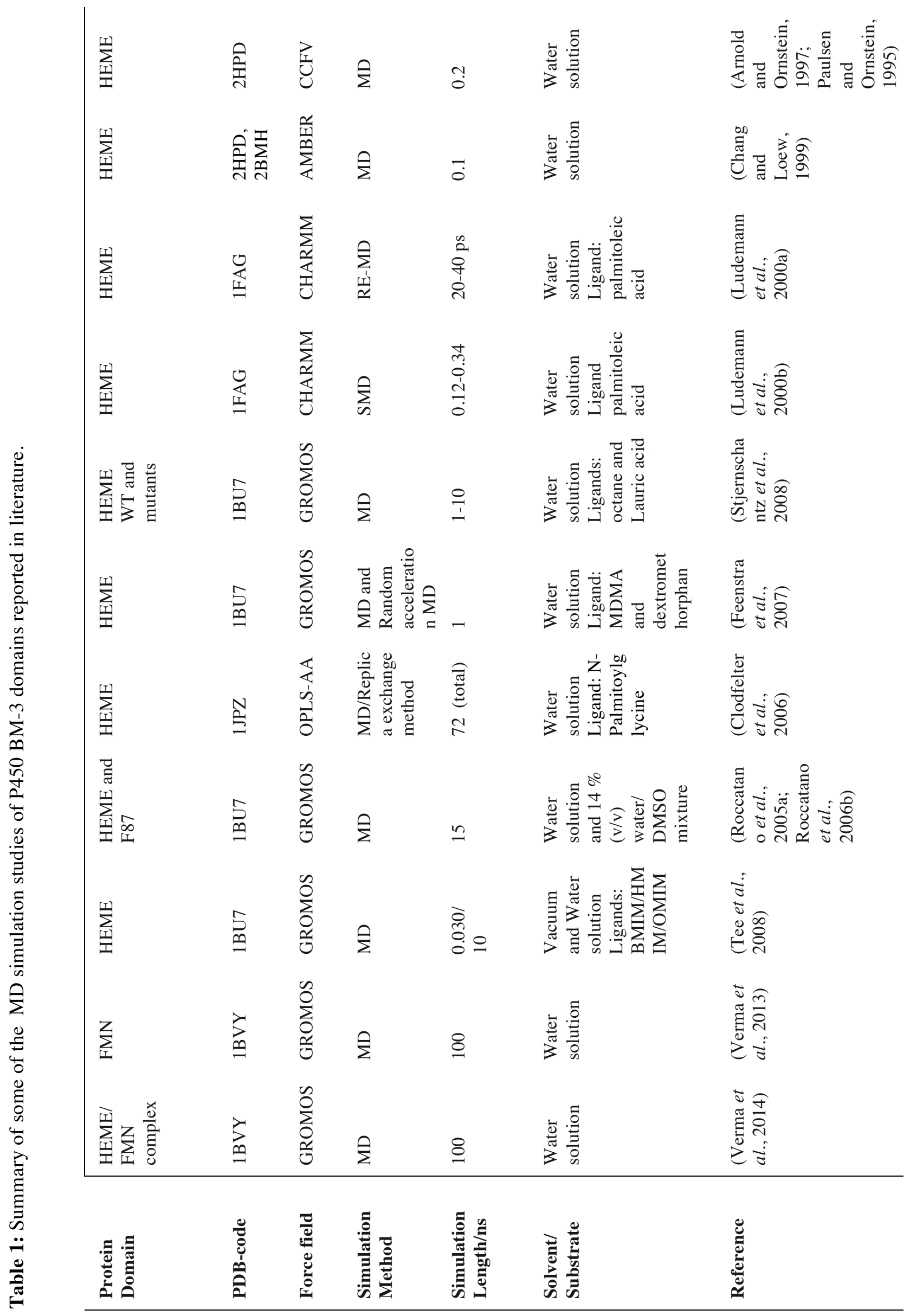


MD simulations studies addressing these points have been focused on disclosing structural and dynamics properties of the enzyme in water and in mixtures of water with cosolvents, with a particular attention to isolated HEME domains and to the FMN/HEME complex. In Table 1, most of MD simulation studies of the P450 BM-3 domain are summarized. In the next paragraphs, I will give an overview of the single domains structure and the review of their computational studies.

\section{The HEME domain}

In Figure 1B, the crystal structure (PDB-code: 1BU7, unit B) of the HEME domain is reported. The HEME domain comprises two major structural subdomains (alpha and beta) composed of fifteen $\alpha$ - and six $3_{10}$-helices (Figure 1B, in blue color), and twelve $\beta$-strands (Figure 1, in red color), respectively. The active site contains the Fe- protoporphyrin IX with the $\mathrm{Fe}$ (III) coordinated with the sulfur atom of the Cys400, and, in the substrate-free enzyme, with a water molecule. The active site is accessible through a substrate access channel (SAC, the silver tubular volume in Figure 1B) and a small water channel. The access mouth of the SAC is located between the F/G loop and the region comprising helix B' and the loop of the first $\beta$-hairpin present in the protein. The change in channel diameter can be easily calculated using CAVER, a program for the calculation of protein internal cavities (Petřek et al., 2006). Given several starting points ( 5 in this case) in proximity of the iron atom, the program searches for channels that emerge outside the protein. By averaging the diameters of the different pathways generated from each starting seeding locations, the average channel diameter profile with respect to the distance from the iron was obtained.

Crystal.Unit A

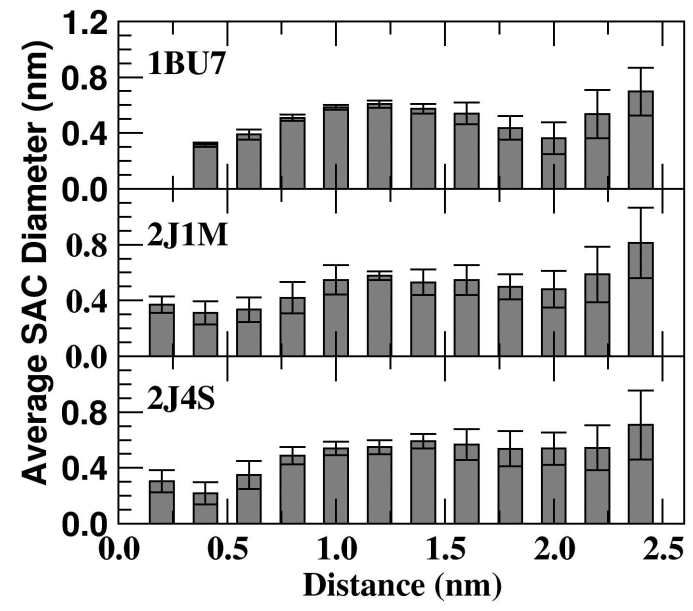

Crystal. Unit B

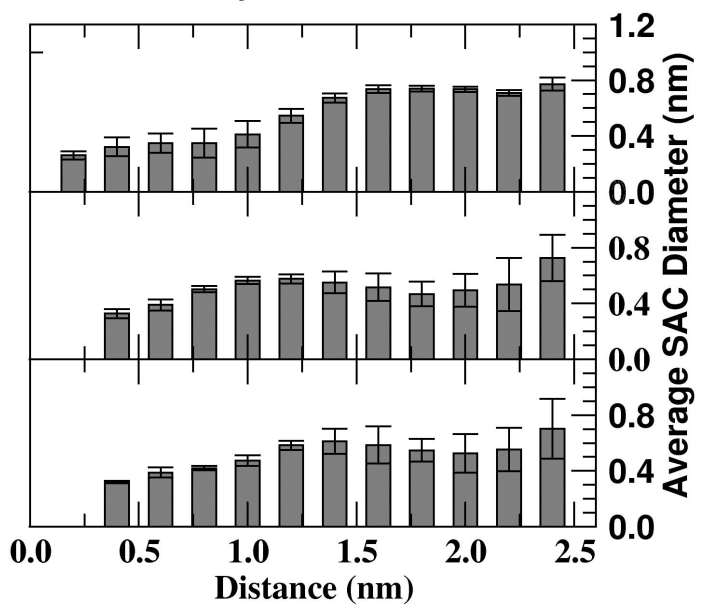

Figure 2: Average SAC diameters as function of the distance from the heme iron on the crystallographic units of three different structure of the HEME domain. 
In Figure 2, average profiles for three different X-ray structures of the domain crystallized in water (PDB-code: 1BU7) and in the presence of two concentration of dimethylsulphoxide (PDB-codes: $2 \mathrm{~J} 1 \mathrm{M}$ and $2 \mathrm{~J} 4 \mathrm{~S}$ ) are shown. The average diameter along the SAC is between $0.43-0.46 \mathrm{~nm}$ with the exception of the 1BU7 unit B structure with a value of $0.51 \mathrm{~nm}$. The SAC increase in diameter going from the heme group to the SAC exit opening (around 2.4 $\mathrm{nm})$ with a maximum $(\sim 0.6 \mathrm{~nm})$ around $1.5 \mathrm{~nm}$. The channel average diameter slighly decreases (with the exception of 1BU7, unit B) around a distance of $2 \mathrm{~nm}$ in correspondence of the SAC entrance. The diameter od the SAC in this region reveals the open/closed state of the SAC in the crystal structure. The size of the channel allows accommodating a large variety of substrates and the enzyme can perform different types of oxydation reactions. It is not clear the function of the enzyme and the nature of the substrate in the B. Megaterium, but it likely consists of polyunsaturated fatty acids (Munro et al., 2002a). However, its substrate specificity has been further expanded by protein engineering with an extended repertoire of regio- and stereo-specific oxidation reactions on a large variety of molecules of industral interest (see also (Whitehouse et al., 2012)).

\subsection{MD studies on the protein dynamics and substrate access channel}

MD simulation studies of the HEME domain started few years later the first crystallographic structure was made available. They were mainly focused on the understanding the conformational change of the protein and on the accessibility of the active site. Although, the time scales of these simulations are outdated for nowadays standards, they have provided a first valuable information on the dynamics of the protein and on the mechanism of substrate binding. The first MD simulation of the P450 BM-3 was published 20 years ago (1995) by Paulsen and Ornstein (Paulsen and Ornstein, 1995). They performed a simulation of 200 ps at $300 \mathrm{~K}$ of the HEME domain whose results confirmed the flexibility of the SAC entrance region. In a second paper, Arnold and Ornstein (Arnold and Ornstein, 1997) have used the previous simulation to addressed the role of the F/G helices in controlling the opening of the mouth region by analyzing the trajectory using a dynamic cross-correlation matrix approach (McCammon and Harvey, 1987). In a second MD simulation study, performed by Chang and Loew few years later (1999), the effect of the substrate on the closure mechanism of the enzyme was also analyzed (Chang and Loew, 1999). The results of this study showed that the binding of palmitic acid into the SAC of the substrate-free open crystal structure, induce its closure by a rotation of the $\mathrm{F} / \mathrm{G}$ helices of the same extended observed in the substrate-bound closed crystal structure. Comparative simulation studies have been performed by the group of Wade to understand the mechanism of substrate expulsion from P450 BM-3 and other types 
of cytochrome P450 (Wade et al., 2004) active sites. In a first study, a method called random expulsion molecular dynamics (REMD) was used to simulate the expulsion of the substrate from the access channel (Ludemann et al., 2000a). In this method a random force is added to the center of mass of the substrate to speed up its diffusion into the active site and to find a way to go out of the protein in a simulation time as short as few nanoseconds. The spontaneous egress of fatty acids substrate, as also of other small molecules, is an event that can require very long MD simulations, likely in the order of several hundred of nanoseconds to microseconds (Feher et al., 1996). For these simulations, the authors have used the CHARMm22 force field (MacKerell et al., 1995). In a second paper (Ludemann et al., 2000b), the same authors have further studied the pathways obtained from the REMD method by steered molecular dynamics simulation method (Isralewitz et al., 2001). They also calculate the free energies involved in the egression mechanism along the different pathways. The results of their simulation evidenced the presence of two possible pathways (named $p w-a$ and $p w-d)$. In the pathway $p w-a$, the substrate diffuse between the FG loop, B' helix/BB' loop and the b1-sheet. In the $p w-d$, it proceed along another exit located between the helices $a$ and $A$. Finally, they have characterized the detailed mechanism of the egression from the main pathway ( $p w-a$, common to other two cytochrome P450cam and P450eryF also considered in this study) showing that the expulsion is accompanied by a large conformational change in the protein backbone, and pointing out the role of the Arg47 at the entrance of the SAC in guiding the negatively charged substrate (pamitoleic acid) in and out the channel (Wade et al., 2004; Winn et al., 2002). Nevertheless, the mechanism of substrate egression proposed in this study does not explain the entrance and egression of other type of substrates. More recently, Stjernschantz et al. (Stjernschantz et al., 2008) have addressed the question for these substrates using the same REMD simulation approach. They proposed the presence of a possible exit route involving the conformational change of the cytochrome P450 in correspondence of one of the propionate group of the heme. The results of this study showed that though this exit pathway is normally accessible for other P450 enzymes (as the human P450 2D6), for the P450 BM-3 was found inaccessible. Clodefleder et al. (Clodfelter et al., 2006) have performed MD simulations using solvent mapping approach to study the flexibility of the active site to accommodate substrates of different types and sizes. In the case of P450 BM-3, the results showed the presence of a high rigidity in the protein structure that make less able to reshape the active site cavity to accommodate large substrate compared to other P450s. Finally, it is worth to mention a theoretical study (not based on MD simulations) by Oprea et al. (Oprea et al., 1997) that evidenced the presence of a water channel in proximity of the heme propionate groups. The water channel was found in different type of P450, and it seems controlled by the conserved Arginine (for P450 BM-3, Arg398) that form 
a salt bridge with one of the heme propionate.

\subsection{MD aided rational design of the active site}

Pleiss et al. have used MD simulations methods to rational design the P450 BM-3 active site and identify potential mutation sites (Pleiss, 2014). They have used amino acid sequences and tridimensional structures of active sites in several cytochromes P450 to obtain sequencestructure-function relationships. In this way, they found that in two substrate-interacting residues (hotspots) only hydrophobic are preferentially observed. Using this information they constructed a minimal and highly enriched P450 BM-3 mutant library by introducing hydrophobic amino acids at the two hotspots Phe87 and Ala328. By screening this library using terpenes substrates, they finally identified variants with either a strong shift or improved regio- or stereoselectivity as compared to P450 BM-3 WT. Finally, they have used simulations to reveal the molecular basis of the observed selectivity. They have carried out several simulations to study the docking modes of terpenes substrates located in the active site of HEME domain variants Ala328Val and Ala328Val/Leu437Phe. The results of these simulations let them identifying potential residues with improved selectivity toward novel substrates (Seifert et al., 2011; Seifert and Pleiss, 2012).

In another study, Feenstra et al. (Feenstra et al., 2007) have rationalized regio-selective hydroxylation of octane and lauric acid by P450 BM-3 wild-type and the three active site mutants Phe87Ala, Leu188Gln/Ala74Gly, and Phe87Val/Leu188Gln/Ala74Gly. In this study they have performed MD simulations starting from proteins conformations with the two substrates modeled with the docking program GOLD 2.0 (Jones et al., 1997) in the active sites. The MD simulations were used to calculate relative free energies for alternate binding orientations of the substrates. Finally, semi-empirical quantum mechanics calculations were used to obtain the activation energies for the rate- limiting $\mathrm{H}$-abstraction. The results of their study further supported the known function of the Arg47/Tyr51 as carboxylic anchoring site. In addition, they evidenced the role of Phe87 in the binding modes of the substrates. Finally, they also found that electrostatic interactions between the protein and the substrate can strongly modulate the substrate's regio-dependent H-abstraction barriers.

\subsection{MD studies on solvent effects}

Solvent molecules play an important role for the structural and dynamical properties of proteins. A major focus of current protein engineering is the development of enzymes that are catalytically active in the presence of organic solvents, and the MD is an important method to unravel the dynamics of protein solvation (Roccatano, 2008). Being the P450 BM-3 a 
promising monooxygenase for biocatalysis applications, several experimental studies have been conducted to understand the inhibitory effect of organic cosolvents on the catalytic mechanism of both the WT protein and its mutants (Kuper et al., 2012a; Kuper et al., 2007a; Seng Wong et al., 2004; Tee et al., 2008). Computational studies have complemented the experimental ones to provide a detailed molecular model of the protein-cosolvent interaction effects. Among the different cosolvents, the attention was mainly focused on the behavior of the HEME domain in aqueous solutions of dimethylsulphoxide (DMSO) (Roccatano et al., 2005a; Roccatano et al., 2006a) and ionic liquids (Tee et al., 2008), respectively. Experimental evidences have shown that there is a considerable difference in the tolerance of the P450 BM-3 WT, and the mutant Phe87Ala mutant in the presence of low concentrations of DMSO and other organic cosolvents (Wong et al., 2004). However, the molecular mechanism of this process was not obvious. In particular, for the mutant Phe87Ala, a substantial lower tolerance than the WT was observed in all the considered cosolvents (Wong et al., 2004). This unusual tolerance of the enzyme is not present in the variant Phe87Ala that rapidly decrease its activity as the concentration of DMSO increases. For instance, at $\sim 14 \%$ (v/v) DMSO, it has a residual activity of only $\sim 30 \%$ whereas the WT in the same conditions retains its full activity (Wong et al., 2004).

The inhibitory effect of the cosolvent on the enzymatic activity is usually associated to the depletion of the functional water layer surrounding the enzyme with the consequent protein denaturation (Roccatano, 2008). Although DMSO, as chaotropic solvents, can induce denaturation by the depletion of the functional water layer surrounding the proteins (Roccatano, 2008), the concentrations used in P450 BM-3 experimental studies are too low to explain its inhibitions as a denaturation effect. Moreover, the unmodified activity of the WT enzyme up to $14 \%(\mathrm{v} / \mathrm{v})$ compared with strongly reduced one of the single mutant suggested that the residue Phe87 is playing a major role in preventing the DMSO inhibition. Indeed, since the Phe87 residue is located in the active site cavity in proximity of the heme (see Figure 1), it was expected that the inhibition effect should be related to the direct interaction of cosolvent molecules with the active site of the enzyme. In addition, the inhibition of the enzymatic activity by different type of cosolvent molecules shows a correlation to their molar volume suggesting a possible size exclusion effect of the cosolvent on the substrate.

To gain insights into the molecular inhibition mechanism, we have performed $15 \mathrm{~ns}$ long MD simulations in pure water and in a 14\% DMSO/water mixture (Roccatano et al., 2005a) using the GROMOS (van Gunsteren et al., 1998) force field with modified heme parameters (Helms et al., 1996). The protein in the two simulations did not show significant variations from the crystal structure due to the presence of cosolvent molecules. However, significant 
differences were observed in regions important for the regulation of the catalytic activity (helices E, F, and G, and in particular in the EF and FG loops) in DMSO/water mixture. In a pure water simulation, the same regions, showed higher mobility but less average deviations from the crystal structure. Interestingly, in both simulations a significant higher mobility was observed in correspondence of the residues Phe42, Arg47, and Tyr51 located at the mouth of the active site (see Figure 1). This high mobility is also evidenced by the high values of the crystallographic B-factors observed in for the different x-ray structures of the protein. During the simulation, DMSO molecules accumulated on the protein surface raising the average local DMSO concentration to 30-40 \% (v/v). They mainly bound in correspondence of the protein surface groves (see the yellow density blobs in Figure 2) to maximize their contacts with the protein, and with a limited discrimination on the chemical nature of the binding amino acid (Roccatano et al., 2006a). The surface absorption reduces the average value of the DMSO diffusion coefficient by $\sim 30 \%$. DMSO molecules accumulated in correspondence of the substrate binding sites in proximity of the SAC entrance (see Figure 3 and Ref. (Roccatano et al., 2005a)) and only one DMSO molecule was found forming hydrogen bonding interactions with the side chain of the residue Arg47 at the end of the simulation. However, even trying to extend the simulation for other $5 \mathrm{~ns}$, and to increase the temperature up to $350 \mathrm{~K}$, the molecule did not further diffuse closer to the active site. In previous MD simulation studies (see above) it was showed that Arg47 play the role of an anchoring and pivoting residue to facilitate the entrance in the SAC of fatty acids substrate. Actually, the mutation of both the residues Arg47 and Tyr51 hamper the binding of fatty acids (Noble et al., 1999; Ost et al., 2000), and the hydroxylation of non-natural substrates (Wong et al., 2005). The result of our simulations suggested that Arg47 play a similar function also for polar molecule (as the DMSO) preventing their rapid diffusion into the active. 


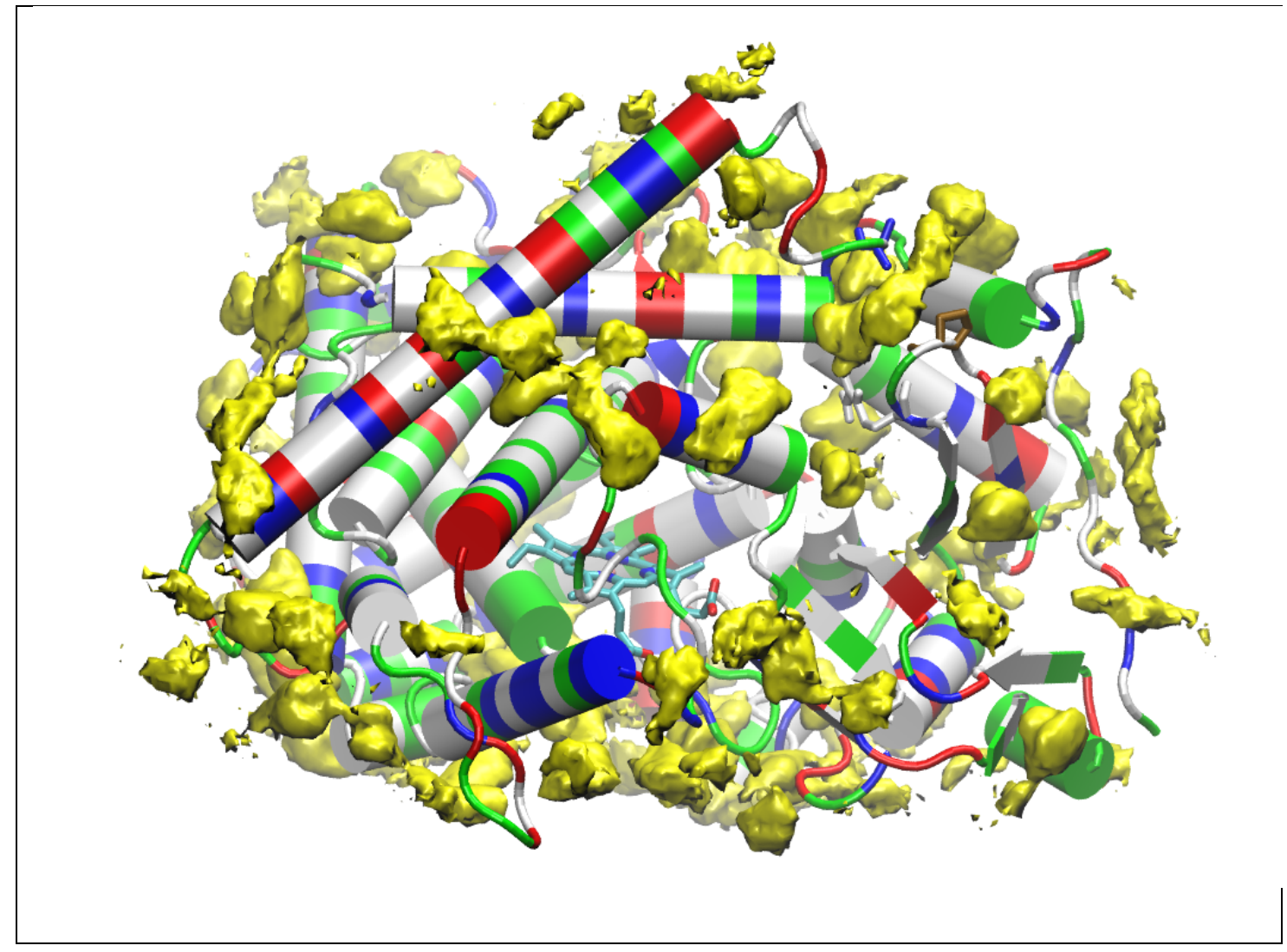

Figure 3: Spatial density distribution of the DMSO molecules around the HEME domain. The distribution was calculated from the last $5 \mathrm{~ns}$ of the trajectory from (Roccatano et al., 2005b) using the GROMACS package. A cubic grid with $0.1 \mathrm{~nm}$ grid spacing was used for all the calculations. The DMSO molecules were centered around the protein molecule in each trajectory frame using a translational-rotational fit to the first configuration of the MD trajectory and removing the periodic boundary conditions. Finally, the atomic positions of the solvent molecules were mapped on a cubic grid centered on the protein and averaged with respect to the number of conformations analyzed. The obtained averaged volumetric density data were visualized using the program VMD (Humphrey et al., 1996). . The protein is represented in cartoon with the amino acids colored according to their chemical nature (red: acid, blue: basic and green: polar, gray: unpolar). The views is oriented as in Figure 1B residues Pro45, Ala191 and Arg47 are represented in liquorice style. The DMSO high density isosurfaces are shown in yellow.

As described before, the opening of the SAC mouth regulates the access of the substrate into the active site. The extent of the opening is usually monitored using the distance between the C $\alpha$ of the amino acids Pro45 and Ala191 (Paulsen and Ornstein, 1995). A survey of the HEME domain crystal structure in the PDB shows that this distance gives a bimodal distribution with the two peaks located at $\sim 0.8$ and $\sim 1.2 \mathrm{~nm}$, respectively. Most of the shorter distances peaks occur in structures with large substrate (e.g. palmitoleate) in the active site, whereas the larger distances are present in substrate-free structures crystallized in water or in the presence of DMSO or other small ligands. The substrate-free structure (PDB-code: 
1BU7), used in the simulations, has two chains in the crystallographic unit cell with C $\alpha$ P45A191 distances equal to 0.87 (in the chain A) and $1.09 \mathrm{~nm}$ (in the chain B), respectively. The simulation in water (using as starting structure the chain A) showed the tendency to further reduce this distance up to $\sim 0.7 \mathrm{~nm}$. The closing of the SAC probably also explains the difficulty to release the substrate from the active site in earlier MD studies (see above). On the contrary, the simulation of the Phe87Ala mutant in the same conditions showed the presence of a bimodal distributions with the two peaks positions located in correspondence of the distances present in crystallographic structures (Roccatano et al., 2006a). In both pure water and DMSO/water mixture simulations, the change of the mouth state occurred within the first $2.5 \mathrm{~ns}$. The HEME domains in the non-stoichiometric complex with the FMN domain (PDB-code: 1BVY) show an extended opening of the SAC mouth with a distance of $\sim 1.6 \mathrm{~nm}$. A very recent simulation study from our group (Verma et al., 2014) of the HEME domain from the complex (chain A) showed that, after the first $20 \mathrm{~ns}$ of simulation, the mount reamin open to a stable distance of $1.1 \pm 0.1 \mathrm{~nm}$. On the contrary, in the simulation of the HEME/FMN complex, the mouth progressively closes during the first $32 \mathrm{~ns}$ to final distance of $0.6 \pm 0.1 \mathrm{~nm}$

MD simulations of the HEME domain (in this case the PDB structure 1BU7) in $14 \%$ $(\mathrm{v} / \mathrm{v})$ DMSO/water mixtures also showed the tendency of the protein to retain the open SAC conformation (Roccatano et al., 2006a). This result results in agreement with the open conformation also present in the crystallographic structures of the HEME domain cocrystallized in DMSO (Kuper et al., 2007b; Kuper et al., 2012b). For the Phe87Ala mutant, the presence of DMSO in the simulations did not changed the shape of the bimodal distribution observed in the water simulation but it induced a widening of the mouth by shifting the two peaks at 1.6 and $1.8 \mathrm{~nm}$, respectively. The bimodal distribution for the P45Ca-A191Ca distance indicates a higher flexibility of the EF helix subdomain in the Phe87Ala mutant compared to the WT. It also suggested that DMSO molecules for the Phe87Ala mutant might have a higher probability to access the active site and, consequently, interfere with the catalytic mechanism. However, to further address this effect on the catalytic mechanism, it was necessary to study a system with DMSO molecules modeled in the SAC, as we will see in the next paragraph.

Another interesting aspect of the SAC gating is the role of the protein collective motions of the protein on its mechanism. A simple way to analyze the structural and dynamical behavior of a protein is the measure of the atomic root mean square deviations (RMSD) and fluctuations (RMSF) defined as (Roccatano, 2007): 


$$
\begin{aligned}
& \operatorname{RMSD}(t)=\sqrt{\frac{1}{N_{A}} \sum_{i}\left(r_{i}(t)-r_{i}^{r e f}\right)^{2}} \\
& \operatorname{RMSF}(i)=\sqrt{\frac{1}{N_{F}} \sum_{t}\left(r_{i}(t)-\left\langle r_{i}\right\rangle\right)^{2}}
\end{aligned}
$$

where the index $i$ indicate the $i$ th atom, $t$ the timeframe along the trajectory, $\mathrm{N}_{\mathrm{A}}$ the total number of atoms considered, $\mathrm{N}_{\mathrm{F}}$ the number of timeframes used, $\mathbf{r}_{\mathrm{i}}(\mathbf{t})$ the coordinates of the $i t h$ atom at time $t$ and $\left\langle\mathbf{r}_{\mathbf{i}}\right\rangle$ its average value, and, finally, $\mathbf{r}_{\mathbf{i}}^{\text {ref }}$ the reference structure, usually the crystallographic one. For clarity, RMSD and RMSF values are normally given per protein residue as average of the atomic values within the residue. In Figure 4, both the RMSD and RMSF of the backbone atoms with respect the starting crystallographic structure of the WT simulations are reported. The highlighted regions, corresponding to the $\alpha$-helices region E-F-G, show the presence of both large deviations and fluctuations, in particular for the DMSO solution simulation (red curves). This behavior is consistent with earlier theoretical analysis (Arnold and Ornstein, 1997), and in agreement with the variations in E-F helices region found among the HEME domain crystallographic structures (Girvan et al., 2004). Furthermore, a similar behavior has been recently observed in longer simulations of the HEME domain as isolated or in the complex with the FMN domain (Verma et al., 2014). All these experimental evidences and simulation results enforce the hypothesis that the motion of this region is involved in ligand gating regulation of the active site of the enzyme (Poulos, 2003).

Recently, the HEME domain has been also studied in the presence of different ionic liquids (IL) as cosolvents. IL comprises a large group of innovative solvents composed by an organic cation (the most common are either the alkyl-substituted imidazolium and the pyridinium cation) and an anion. They are not flammable, thermally stable with negligible vapor pressure, which makes them interesting from safety and environmental standpoints. Moreover, their physico-chemical properties (for example polarity, hydrophobicity or miscibility) can be tailored to specific applications. These capabilities are particularly attractive for biocatalysis applications to overcome environmental and safety hurdles associated with the use of conventional organic solvents (van Rantwijk et al., 2003). 


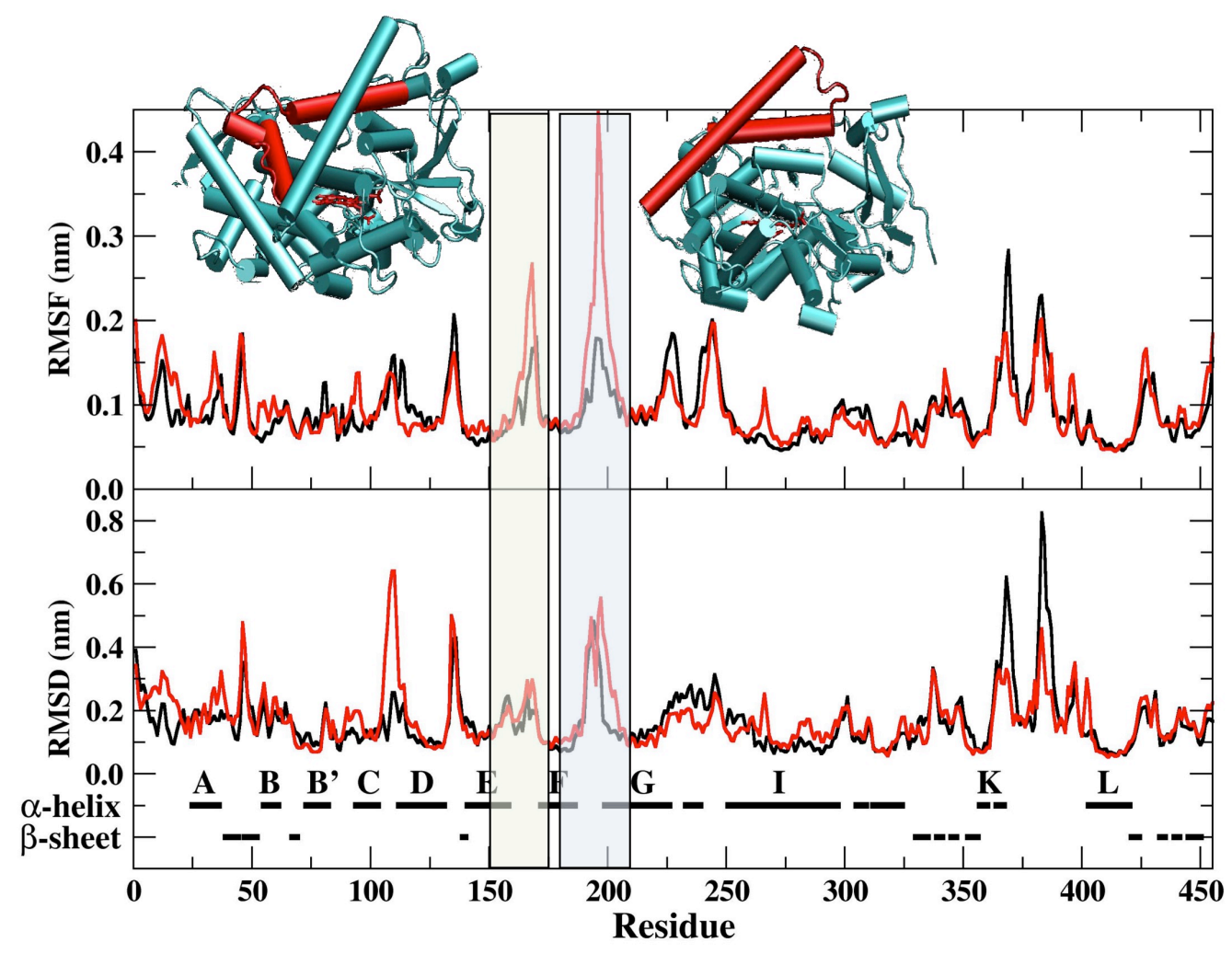

Figure 4: Backbone RMSD (bottom graph) and RMSF (top graph) per residue of the WT simulations in water (black) and in $14 \%(\mathrm{v} / \mathrm{v}) \mathrm{DMSO} /$ water (red), respectively. The RMSD curves are calculated with respect the crystallographic structure. The bars at the bottom indicate the locations of the secondary structure elements in the crystal structure of the P450 BM-3. The two shaded rectangular regions, and the protein structures on the sides, highlight the EF and FG subdomains regions.

In a recent paper, we have studied the effect of different ILs on the P450 BM-3 enzymatic activity (Tee et al., 2008). For these ionic liquids, an inhibition effect on the protein correlated to the length of the hydrophobic chain in the order of 1-ethyl-> butyl- > hexyl- > octyl- has been observed (Tee et al., 2008). For the 1-butyl-, 1-hexyl-, octyl-3methylimidazolium ions (see Figure 5A), we have performed MD docking simulations to give a qualitative molecular model of the IL interaction with the HEME domain. For ILs located in proximity of the entrance to the SAC, results showed that their positively charged head groups interact with the negative Glu13 (see Figure 3 in (Tee et al., 2008)). This residue is located in proximity of the entrance to the SAC (see Figure 5C) and it belongs to the substrate recognition region that is believed to be important for initial binding of natural fatty acid substrates (Ravichandran et al., 1993). The binding of IL molecules to this region can interfere with the substrate access to SAC and leads to the observed increase of $\mathrm{Km}$ values (Tee et al., 2008). In a MD simulation of the HEME domain (see Table I) in 0.2 M 1-butyl-3- 
methylimidazolium/water solutions, recently performed in my group, the final distribution of the IL was consistent with the MD docking study in vacuum. Figure 5B show two view of the spatial density distribution around the protein calculated from the last $5 \mathrm{~ns}$ of this simulation. The yellow density blobs show that the IL bound mainly in correspondence of negatively charged amino acids (aspartic and glutamic acids) on the protein surface (shown in red in Figure 5B). Finally, in Figure 5C, the detail of spatial density distribution in correspondence of the SAC entrance (residues 45, 191) shows the accumulation of the IL around the Glu13. The contact between the Ala191 and Pro45 indicates that the enzyme is in the closed conformation at the end of this simulation and thus IL molecules were not able to enter into the SAC.

\subsubsection{Behavior of solvent molecules in the active site}

In order to study the effect of DMSO in the active side of the HEME domain, we have also simulated both the WT and the Phe87Ala mutant HEME with DMSO molecules inserted in substrate channel. As mention before, the Phe87Ala mutant exhibits an improved regionselectivity and substrate specificity compared to WT but it has a significant lower tolerance toward DMSO (Wong et al., 2004). The substitution of the bulky phenylalanine with an alanine in the active site suggested that the inhibition mechanism is related to the interaction of DMSO molecule with this residue. In particular, in the active site it could interfere with the catalytic mechanism either by producing a steric hindrance that restricts the accessibility of the substrate or by the direct replacement of the water coordinating the heme iron. To verify the two inhibition mechanism hypotheses, DMSO molecules were randomly placed in the $\mathrm{SAC}$ of an equilibrated protein conformation from previous simulations. By placing one and three molecules, respectively, and assuming an average number of 20 water molecules in the channel, concentrations of 18 and 42\% (v/v) DMSO/water, respectively, were obtained. Hence, from these starting conformations, two $7 \mathrm{~ns}$ long simulations were performed and compared with the unmodified $15 \mathrm{~ns}$ long reference ones (Roccatano et al., 2006a). The analysis of the trajectories showed that the presence of DMSO molecules in the active site did not change the overall structural properties of both WT and Phe87Ala mutant, but it strongly interfered with the iron coordination of water molecules. The displacement of the water molecule from the iron plays an important role in triggering the electron transfer from the reductase part of the monooxygenase. In fact, the absence of the coordinating water molecule increases the redox potential of the iron triggering its reduction by the electrons from the FMN domain (Haines et al., 2001). Therefore, DMSO molecules by destabilizing the coordinating water molecule can induce a premature electron transfer to the iron that would interfere with the substrate induced catalytic activation of the enzyme (Haines et al., 2001). In 
all the simulations, the covalent water-iron coordination bond was roughly modeled using electrostatic and Lennard-Jones interactions.

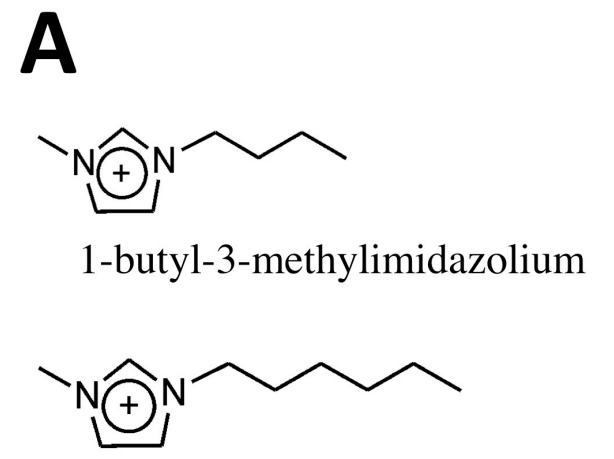

1-hexyl -3-methylimidazolium<smiles>CCCCCCCCn1cc[n+](C)c1</smiles>

1-octyl-3-methylimidazolium

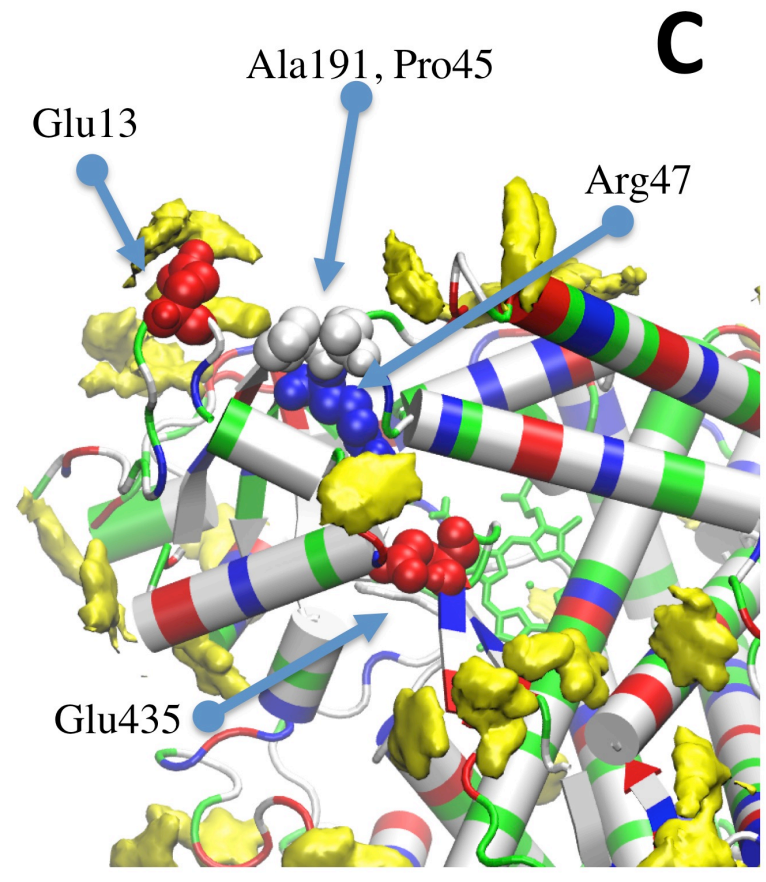

B
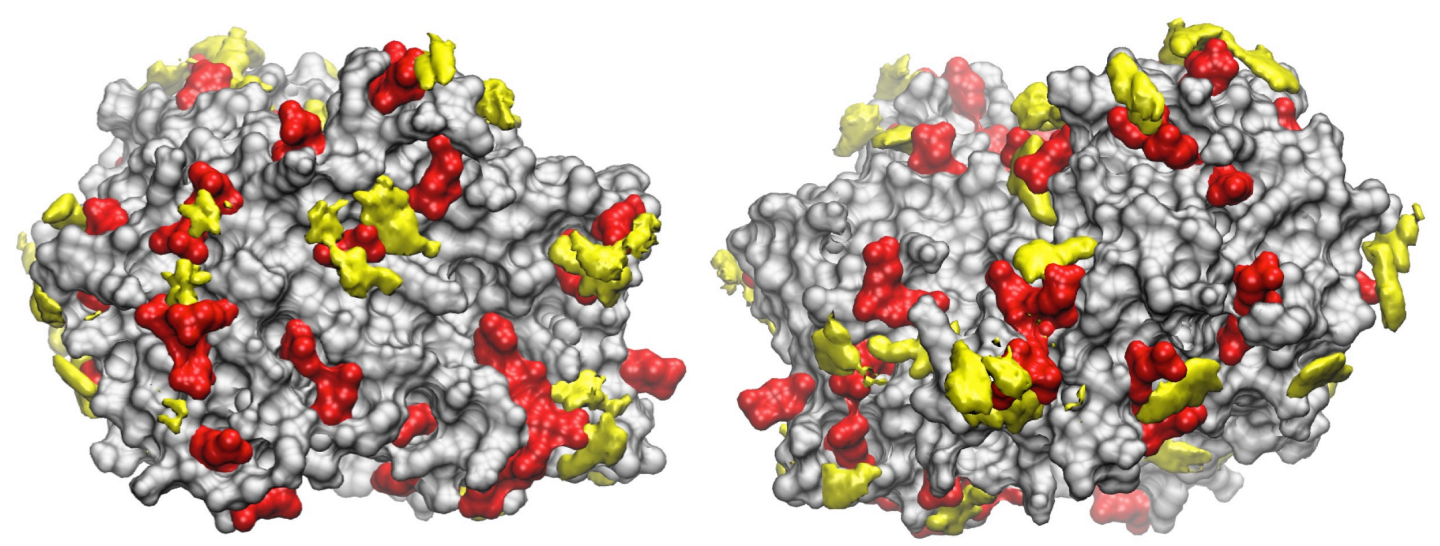

Figure 5: A. The three ILs used in the MD docking study with the HEME domain. B. Two view of the spatial density distribution of 1-butyl-3-methylimidazolium (in yellow) on the HEME domain surface. The MD simulation was perfromed using the GROMOS96 (van Gunsteren et al., 1998) force field. The spatial density distribution was calculated from the last $5 \mathrm{~ns}$ of the trajectory using the same procedure described in the caption of Figure 3. C. Detail of the spatial density distribution in correspondence of the substrate access channel. The secondary structure elements of the protein is represented in cartoon style. Some relevant amino acids are indicated by the arrows and the atoms represented as van der Waals sphere. 
Therefore, in the water simulation, a water molecule is firmly coordinated to the iron to an average Fe-Ow distance slightly longer $(\sim 0.3 \mathrm{~nm})$ than the one observed in the crystallographic structure (1BU7, $0.26 \mathrm{~nm})$. However, for the purpose of the study, the accuracy of this value is less relevant than the modeling of a stable Fe-Ow coordination bond. In the simulation with one DMSO in the active site the distance remain the same for the WT but in the case of the Phe87Ala mutant the DMSO molecule get closer to the iron and eventually displaces the coordinating water molecule. At higher DMSO concentration, the water molecule in the WT simulation continues to coordinate the iron for most of the simulation time. On the contrary, for the Phe87Ala simulation in the same conditions, a DMSO molecule displaces the water molecule and remains at a distance of $0.45 \mathrm{~nm}$ to the iron for a considerable fraction of the simulation time (Roccatano et al., 2006a). For steric hindrances with residues the Thr268 and Ala264, the DMSO molecules could not occupy the space above iron to form a coordination bond with the sulfur or oxygen atom. However, the DMSO molecule approached the iron so closely that it was expected that such reaction might also occur upon a further rearrangement of the active site residues, not observed in this short simulation. This result showed that DMSO could interfere with the iron coordination of the water molecules, in particular for the Phe87Ala mutant due to the absence of the steric hindrance from the bulky phenyl group. Therefore, it gave a qualitative suggestion on the possible role of DMSO molecules in reducing the catalytic activity of Phe87Ala. These findings have been recently confirmed by the determination of both WT and Phe87Ala mutant HEME domain crystal structures permeated with DMSO (Kuper et al., 2012a; Kuper et al., 2007a). The crystals of the proteins were diffracted after soaking them in $14 \%(\mathrm{v} / \mathrm{v})$ and $28 \%(\mathrm{v} / \mathrm{v}) \mathrm{DMSO} /$ water solutions. The WT crystal structures, at both low- (PDB-code: 2J4S) and high- (PDB-code: 2J1M) DMSO concentration, do not show large conformation change compared to those previously solved in water buffer. The 2J4S structure shows a nonplanar distortion of heme and the iron coordinating water molecules displaced to a distance of $0.377 \mathrm{~nm}$. On the contrary, the water molecule in the $2 \mathrm{~J} 1 \mathrm{M}$ structure is replaced by one of DMSO, and anomalous scattering measurements confirmed that this molecule coordinates the iron with its sulfur atom (Kuper et al., 2007a). As expected, the presence of the bulkier DMSO induces larger changed in the active site, as for example, by increasing the I-helix kinking. Also the Phe87Ala mutant of the HEME, crystallized and diffracted at the same DMSO concentrations of the WT (14\% (v/v): PDB-code: 2X7Y; 28 (v/v): PDB-code: 2X80) retain a similar structure as in just water solutions, though, at higher DMSO concentration larger RMSD deviations (Kuper et al., 2012a). In both structures, a DMSO molecule was found in the active site with Fe- $\mathrm{O}_{\text {DMSO }}$ distances of 0.325 and $0.303 \mathrm{~nm}$ for the $2 \mathrm{X} 7 \mathrm{Y}$ and 2X80, respectively. In both cases, the DMSO molecule was found to be oriented towards the 
heme iron but not coordinating it. At low concentration, a water molecule was found in proximity of the iron denoting a dynamic coordination process between the two solvent molecules (Kuper et al., 2012a). On the contrary, at high DMSO concentration, no water molecule was found in proximity of the iron atom. These crystallographic data are also in agreement with results of previous MD simulations and experimental measurements (Jovanovic and McDermott, 2005) on the role of the residue Phe87 as gatekeeper to the access of the heme. Specifically, at low $(<14 \%$ (v/v) DMSO/water concentrations, the frequency and residence of DMSO molecules in the SAC is low enough that allow the phenyl group to impede DMSO molecules the displace the water molecules from the coordination site of the iron atom. As the concentration DMSO concentration increase the frequency of DMSO entering the SAC increase, and so also the probability of displacement of the water coordinating the iron. In the case of Phe87Ala variant, the side chain methyl group of the Alanine 87 is not able to efficiently protect the active site from DMSO molecules interference even at low cosolvent concentrations. However, more accurate modeling studies are necessary to fully understand the active site dynamics and thermodynamics of DMSO/water competition and binding. It is likely that this mechanism is common to other organic solvents that show a similar effect on the activity (Wong et al., 2004).

Finally, I will conclude this part by mentioning the main result of our modeling study of the IL in the SAC channel (Tee et al., 2008). The MD docking simulations with IL cations modeled inside the SAC resulted in their positively charged head groups interacting with the residue Glu435 and with the IL tails oriented towards the heme (see Figure 3 in (Tee et al., 2008)). Since the Glu435 residue is located in proximity of the access channel entrace, this result suggested that ILs could interfere with the access of the substrate in the active site. However, it will require more extensive experimental and theoretical studies to prove this hypothesis, and, to elucidate the inhibition mechanism of these ionic liquids.

\section{FAD/NADPH and FMN reductase domains}

The structure of the FAD/NADPH domain (PDB-code: 4DQK see Figure 1A) was recently solved (Joyce et al., 2012). Different homologous structures of the eukaryotic cytochrome P450 reductase (CPR) are available in the PDB (e.g. PDB-code: 1AMO). This complex has been recently studied using NMR and x-ray scattering methods and the results of these studies provide important information to understand binding modes and dynamics of the two domains (Huang et al., 2013). To the best of our knowledge, MD simulation studies of the P450 BM-3 FAD domain have not yet reported in the literature. 
The FMN domain serves as one or two electrons donor to the heme iron (Hazzard et $a l .$, 1997). The FMN cofactor switches between the fully oxidized and the semiquinone state during the catalytic turnover. Contrarily to other $\mathrm{P} 450$ enzymes in which $\mathrm{FMNH}_{2}{ }^{2-}$ hydroquinone is the reduction species, the heme iron of P450 BM-3 is reduced via a thermodynamically unstable $\mathrm{FMNH}^{-}$semiquinone (Hazzard et al., 1997; Sevrioukova and Peterson, 1996; Sevrioukova et al., 1996a). In the absence of a substrate, the FMN cofactor, locked in a thermodynamically stable hydroquinone state, is not able to transfer electrons to the heme iron (Sevrioukova et al., 1996a). This peculiar and unique mechanisms produce an higher turnover rate than other cytochromes of the P450 family (Narhi and Fulco, 1987b). The molecular environment surrounding the FMN cofactor in its domain has a strong influence on this mechanism by modulating its redox potential. Mutagenesis experiments on the amino acids of FMN binding loops have shown that this region is responsible for stabilizing the different redox states of FMN cofactor (Narhi and Fulco, 1987b; Pylypenko and Schlichting, 2004). The insertion of a glycine residue in the re-face (inner) loop resulted in the stabilization of the neutral semiquinone state as observed in other diflavin reductases (Chen and Swenson, 2008).

\subsection{MD simulation studies of the FMN domain}

The effect of FMN cofactor and its redox states (oxidized and reduced) on the structure and dynamics of the FMN domain was recently studied by our group using MD simulations (Verma et al., 2013). The protein with and without FMN cofactor shows significant differences in the amplitudes of the atomic fluctuation. The reduced FMN state was found to affect the overall structure and dynamics of protein. The conformation of the si-face FMN binding loop (L $\beta 3$ ) was influenced by the change, upon reduction, of the protonation state of FMN cofactor or by the absence of the FMN cofactor in the apo-protein. In the last case, the overall structure of the protein remains quite close to the crystallographic (holo) one but large deviations and fluctuations are observed in correspondence of the FMN binding loops (Verma et al., 2013). The high flexibility of this region could explain the easy rebinding of the FMN cofactor as observed in experimental studies (Kasim et al., 2009; WittungStafshede, 2002). The dynamics of the protein was also analyzed using the principal component analysis (Amadei et al., 1993; García, 1992) to characterize the collective modes of backbone atoms. For both the holo- and apo-proteins, the main collective modes are dominated by the fluctuations of two loops, $L \beta 2$ and L $\alpha 2$, both located in the opposite site of the FMN binding pocket. The inner FMN binding loop (L $\beta 3$ ) contributes to the most important principal mode of FMN domain in the oxidized state, whereas the outer one (L $\beta 4$ ) 
contributes to first principal mode in the reduced state. These different dynamic behaviors of the domain in the two-oxidation states plays an important role in the reorientation of the FMN domain with respect the FAD and in controlling the mechanism of electron transfer to the HEME domains. In fact, recent studies have shown for the homologous eukaryotic CPR a redox-linked domain movements in its catalytic cycle (Huang et al., 2013). Finally, we also observed that the FMN cofactor during the simulations adopts different conformations due to the large motion of its flexible ribityl chain. The binding region of the ribityl chain is the most conserved region of the protein since it plays an important role in controlling the cofactor binding. The FMN in the oxidized state adopts a large variety of conformations state compared to the reduced one as consequence of the changing in the hydrogen-bonding network of the two states. The dynamics of the isoalloxazine ring is dominated by the first 8 principal modes. With the exception of the first modes, all the other seven are identical for both redox states. In oxidized state, the isoalloxazine remains almost planar (average bending angle of $177^{\circ}$ ) whereas in reduced state, it bends of $17^{\circ}$ (average bending angle of $160^{\circ}$ ) along the N5-N10 axis. The bending of the isoalloxazine ring along this axis resulted in the socalled butterfly mode as the dominant vibrational motion of the ring structure.

\section{The HEME/FMN complex}

The crystal structure of the complex between HEME and the FMN domains (PDB-code: 1BVY) was solved at the end of the "90 (Sevrioukova et al., 1999). This structure is a ternary complex with two HEME molecules bound to one FMN domain. In the crystal structure of the rat CPR (PDB-code: 1AMO) the FMN domain shows a different orientation with respect the one found the in FMN/HEME complex (Munro et al., 2002b). This orientation raised concerns about the validity of the latter x-ray structure as a accurate model for the interaction between the FMN and HEME domain in the complete P450 BM-3 structure. However, both the recent experimental evidences of the large domain rearrangements observed in the eukaryotic CPR (see Figure 5 in Ref. (Huang et al., 2013)) and the dimeric biological assembly of the P450 BM-3 in solution (Black and Martin, 1994; Neeli et al., 2005) give support to the idea that the relative orientation of the two domains in 1BVY are consistent with a flexible model of the whole protein (Girvan et al., 2011).

\subsection{MD simulation studies of the complex}

Although the FMN and HEME domain bind in a reliable relative orientation in the crystal complex, atomic distances among its fmn-heme cofactors indicate that the two domains are 
not in a competent arrangement for an efficient electron transfer (Munro et al., 2002b). In order to better understand the interactions and arrangements of the FMM and HEME domain in solution, we have recently performed MD simulations in water of the isolated domains and of their complex (Verma et al., 2014). The structures of the two domains do not show significant differences from crystallographic one in $100 \mathrm{~ns}$ of MD simulations. In the isolated HEME domain, the major conformational changes were observed in the FMN binding region especially in C helix and H/I and K/L (residue 385 - 395) loops. Conversely, the complex undergoes an inter-domain conformational rearrangement in the first $10 \mathrm{~ns}$ of the simulation that increases its compactness (the radius of gyration changes from $2.42 \mathrm{~nm}$ in the crystal structure to $2.33 \mathrm{~nm}$ ) and reduces of $22 \%$ the inter-domain average minimum distances (from $1.81 \mathrm{~nm}$ in the crystal structure to $1.41 \pm 0.09 \mathrm{~nm}$ during the simulation).

In this study, we have tried to understand how this rearrangement affects the ET rate between the two domains. The ET between the FMN and the HEME cofactors was studied using the approach proposed by Beratan et al. (Beratan et al., 1991), and implemented in the Pathways program (Balabin et al., 2012). For a given protein conformation, the program identifies an effective ET coupling by evaluating the highest electronic tunneling coupling $\left(\mathrm{T}_{\mathrm{DA}}\right)$ through bonds and space between a donor and the acceptor (Beratan et al., 1991). The Pathways program identify a series of consecutive interatomic distances (pathways) from a given electron donor atom to an acceptor that maximize the value of $\mathrm{T}_{\mathrm{DA}}$ by assigning different contributions whether the distance represents a covalent bonds $\left(\varepsilon^{\mathrm{cb}}\right)$, hydrogen bonds $\left(\varepsilon^{\mathrm{hb}}\right)$ or through space jump $\left(\varepsilon^{\mathrm{sj}}\right)$. The contributions are defined by the following empirical expressions:

$\varepsilon_{i}^{c b}=0.60$

$\varepsilon_{j}^{h b}=0.36 e^{-1.70(R-2.80)}$

$\varepsilon_{k}^{s j}=0.60 e^{[-1.70(R-1.40)]}$

where R (in Ångstroms) is the distance between two atoms in the path segment. Hence, the value of $\mathrm{T}_{\mathrm{DA}}$ is calculated as proportional to the product of all the contributions:

$$
T_{D A} \alpha \prod_{i} \varepsilon_{i}^{c b} \prod_{j} \varepsilon_{j}^{h b} \prod_{k} \varepsilon_{k}^{s j}
$$

The ET pathways between FMN/HEME were calculated on the crystal structure, and on different conformations sampled along the MD trajectory. For the calculations, the C8 or C7 atom of the FMN isoalloxazine ring, and the HEME iron have been used as donors and 
acceptor, respectively. Finally, the non-adiabatic ET reaction rate $\left(\mathrm{K}_{\mathrm{ET}}\right)$ was estimated using the equation (Marcus and Sutin, 1985):

$K_{E T}=\frac{2 \pi}{\hbar} \frac{e^{\frac{\left[-(\Delta G+\lambda)^{2}\right]}{4 \lambda k_{B} T}}}{\sqrt{4 \pi \lambda k_{B} T}}\left|T_{D A}\right|^{2}$,

where $\Delta G$ is the driving force and $\lambda$ is the Marcus' reorganization energy for the ET reaction, $\hbar=h / 2 p$ with $h$ the Plank constant, and $\mathrm{k}_{\mathrm{B}}$ is the Boltzmann constant. The difference in the reduction potential of the FMN and HEME cofactor $(-0.224 \mathrm{eV}$ ) was used as $\Delta G$ (Munro et al., 1996; Munro et al., 2002a; Page et al., 1999). The value of the reorganization energy $\lambda$ was considered equal to $0.7 \mathrm{eV}$ as a good approximation of the inter protein ET (Moser et al., 1992; Munro et al., 2002a; Page et al., 1999).

The simulations evidenced a conformation arrangement that changed the average distance between the FMN and HEME domains. This result confirms the previous theoretical hypothesis supported by experimental observations (Aigrain et al., 2012; Munro et al., 2002a; Sevrioukova and Peterson, 1996) of the non-competent arrangement for an efficient ET of the crystallographic structure of the complex. The conformations obtained from the simulation present pathways with shorter distances between the two domain's cofactors that give ET rates consistent with available experimental data (Sevrioukova et al., 1996a). In Figure 6, the graph of the pathways among the amino acids calculated from conformations extracted every $5 \mathrm{~ns}$ in the last $50 \mathrm{~ns}$ of the simulation and the most effective one observed in the crystal structure are reported. The rearrangement of the two domains create new contacts not observed in the crystal structure that shortcuts the distance between the iron and the FMN cofactor. The average values of $\mathrm{K}_{\mathrm{ET}}$ calculated from some the new ET pathways have the same order of magnitude of those obtained from experimental measurements (Verma et al., 2014).

The principal component analysis of the trajectories has also shown a clear correlation between collective modes of the complex, and the selective activation of different ET pathways along the trajectory (Verma et al., 2014). We believe that inter- and intradomain motions in the complete enzyme play an important role in the functionality of the enzyme by modulating the ET rate. The results of this theoretical study are consistent with the available experimental data, and provide further insight at the atomistic level to understand the structure and dynamics of this complex enzyme. In particular, structural determinants of the inter-domain ET mechanism can suggest important information to extend the knowledge of the P450 BM-3 enzyme for a better exploitation in biotechnological applications. In fact, this study have identified several key amino acids (see Figure 6) involved in the ET tunneling from FMN to HEME cofactor. These amino acids can be specific targets of site directed 
evolution experiments to prove their importance in the ET mechanism. However, only the reconstruction of the complete structure of the enzyme will disclose all the secrets of its complex modus operandi.
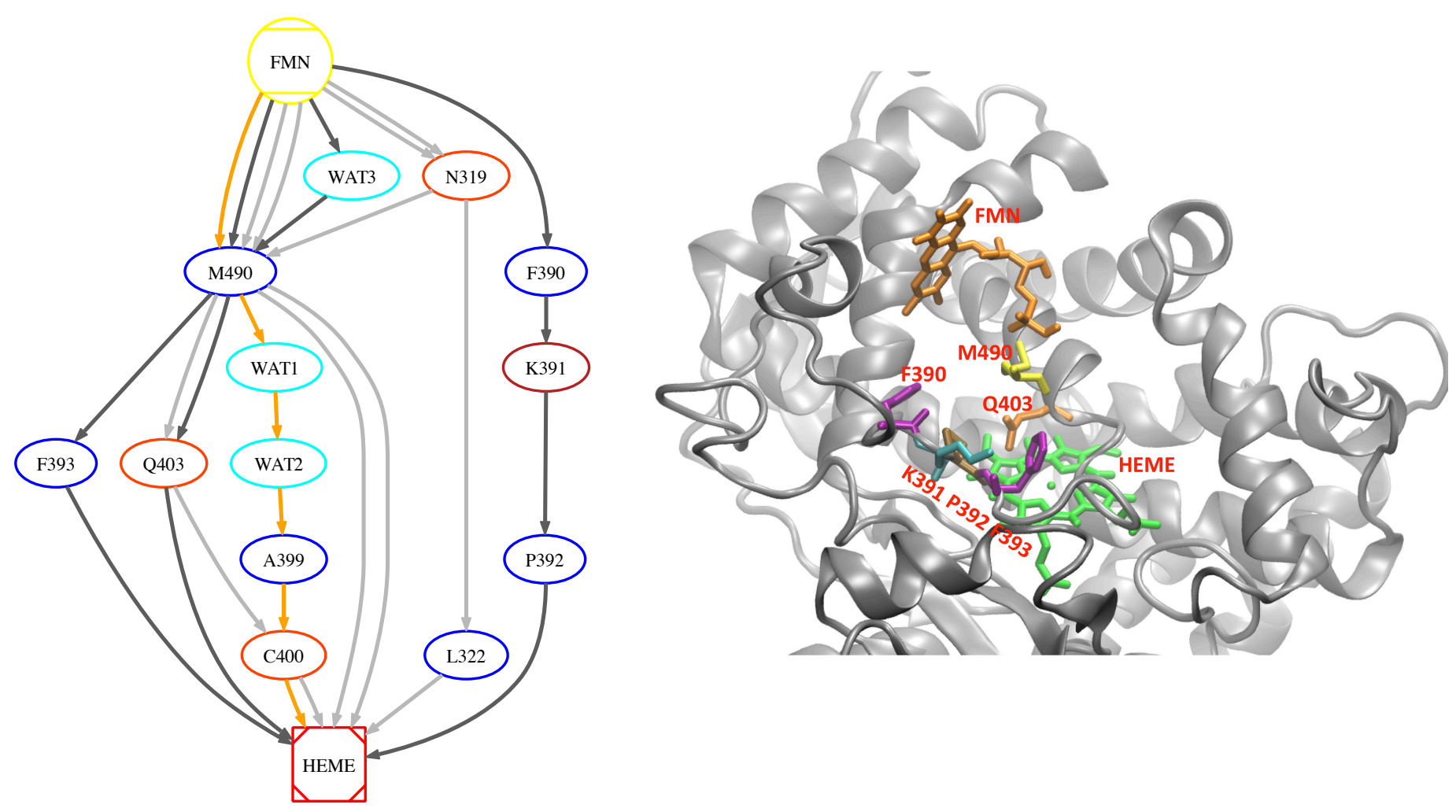

Figure 6: Left panel. ET pathways in the FMN/HEME complex. The pathways in grey colours are the one obtained from the analysis of the simulation. The darker indicate the most relevant ones with calculated $\mathrm{k}_{\mathrm{ET}}>10 \mathrm{~s}^{-1}$. The orange pathway was obtained from the crystallographic structure. The nodes of the graph indicate the amino acids and the water molecules in the pathways. Right panel. Detail of crystal structure of the complex at the end of 100 ns simulation. For clarity, only the FMN group of the FMN domain is showed. The amino acids involved in the most important ET pathways are represented in liquorice.

\section{Summaries and Outlook}

In this topical review, we have reported the major results in the study using MD simulations of the domain of the monooxygenases P450 BM-3. These simulations have addressed many structural, and dynamics aspects of this enzyme trying to clarify the molecular mechanism of its function. Within the limit of the classical MD simulations, these studies have provided 
interesting results to predict the interaction of the protein with cosolvents and substrates, give an accurate representation of the domain dynamics, and its influence in the ET transfer between them. These results have just scratched the surface of the dynamics of this complex enzyme and other puzzling aspects are still waiting to be explored with future simulations. I expect that the extension of simulations on microseconds time scale, the use of multiple trajectories and improved force fields will provide a model of the opening/closing mechanism of the HEME domain and its effect on the diffusion of the substrate or cosolvents molecules in the active site. Moreover, the use of thermodynamics calculation methods (as, for example, umbrella sampling (Berendsen, 2007)) can be used to quantify the energy involved in this process. Long time scale simulations can also provide a better understanding of the effect of solvents and cosolvent on the structure and dynamics of the enzyme. In addition, molecular details of the enzyme interaction with electrodes (surfaces), polymers or electron mediator molecules have important impacts in biotechnology and medicine but not yet surveyed using MD simulations. In this directions, we have recently completed a study on the interaction of the electron mediator Cobalt(II) Sepulchrate with the HEME domain and its HEME/FMN complex (Rajini Verma, PhD thesis, 2013). The results of this study report for the first time the effect of the electron mediator on the enzyme stability, the distribution of the binding sites and their role on the ET mechanism.

On the long term, the most important and challenging milestone to reach in the theoretical study of this enzyme is the building of a reliable model of the full enzyme in its dimeric form that satisfies all the biochemical and biophysical data available. The most recent biochemical and mutagenesis studies (Girvan et al., 2011) of the ET pathways in the enzyme provide clues that the dimeric arrangement of enzyme domains follows the scheme shown in Figure 7. In this scheme, the reductase domains are electronically coupled within the same protein chain (same color) but then there is a cross link and the electrons (blue lines) converge to the active site of the HEME domain in the other chain.

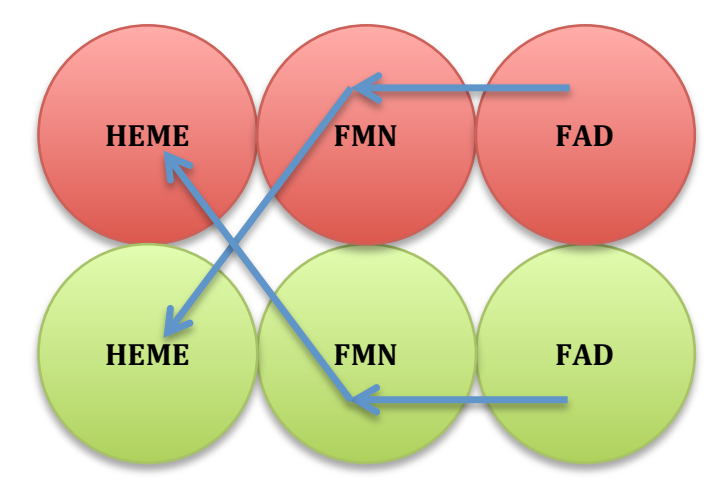

Figure 7: Diagram showing the possible arrangment of the domains in the dimeric structure of the P450 BM-3 based on the intra domain ET. 
It will be very interesting to learn how this process is connected with the actual tridimensional assembly of the dimer and with its dynamics. In this respect, our recent approach to study the dynamics of domain interaction can be a useful to prove the quality of the assembly models by calculating the topology and properties of their ET pathways network. Still, the MD study of single domains and in particular the HEME can reveal many aspect concerning the effect of mutations on the protein functionality. Beside it has been observed changes in the protein specificity with mutations involving amino acids not directly located in the active site (Whitehouse et al., 2012). These effects can be related to changes in structure that can be observed by X-ray diffraction and/or in enzyme dynamics, in the last case MD simulations and possible NMR studies can provide valid tools to further explore the dynamics of this complex enzyme.

\section{Acknowledgements}

The author thanks all collaborators in the research on P450 BM-3 and, in particular, Prof. U. Schwaneberg (RWTH Aachen, Germany) and Dr. T. S. Wong (University of Sheffield, UK) for introducing him to this amazing enzyme.

\section{References}

Aigrain L, Fatemi F, Frances O, Lescop E and Truan G 2012 Dynamic control of electron transfers in diflavin reductases Int J Mol Sci 13 15012-41

Amadei A, Linssen A B M and Berendsen H J C 1993 Essential dynamics of proteins Proteins: Struct., Funct. and Gen. 17 412-25

Arnold G E and Ornstein R L 1997 Molecular dynamics study of time-correlated protein domain motions and molecular flexibility: cytochrome P450BM-3 Biophysical journal 73 1147-59

Balabin I A, Hu X and Beratan D N 2012 Exploring biological electron transfer pathway dynamics with the Pathways Plugin for VMD J Comput Chem 33 906-10

Beratan D N, Betts J N and Onuchic J N 1991 Protein electron transfer rates set by the bridging secondary and tertiary structure Science 252 1285-8

Berendsen H J C 2007 Simulating the physical world : hierarchical modeling from quantum mechanics to fluid dynamics (Cambridge ; New York: Cambridge University Press)

Black S D and Martin S T 1994 Evidence for conformational dynamics and molecular aggregation in cytochrome P450 102 (BM-3) Biochemistry 33 12056-62

Chang Y T and Loew G H 1999 Molecular dynamics simulations of P450 BM3--examination of substrate-induced conformational change Journal of biomolecular structure \& dynamics 16 1189-203

Chefson A and Auclair K 2006 Progress towards the easier use of P450 enzymes Mol Biosyst 2 462-9

Chen H C and Swenson R P 2008 Effect of the Insertion of a Glycine Residue into the Loop Spanning Residues 536-541 on the Semiquinone State and Redox Properties of the Flavin Mononucleotide-Binding Domain of Flavocytochrome P450BM-3 from Bacillus megaterium Biochemistry 47 13788-99

Clodfelter K H, Waxman D J and Vajda S 2006 Computational solvent mapping reveals the importance of local conformational changes for broad substrate specificity in mammalian cytochromes P450 Biochemistry 45 9393-407 
Di Nardo G and Gilardi G 2012 Optimization of the Bacterial Cytochrome P450 BM3 System for the Production of Human Drug Metabolites Int J Mol Sci 13 15901-24

Dror R O, Dirks R M, Grossman J P, Xu H F and Shaw D E 2012 Biomolecular Simulation: A Computational Microscope for Molecular Biology Annu Rev Biophys 41 429-52

Estabrook R W 2003 A passion for P450s (rememberances of the early history of research on cytochrome P450) Drug metabolism and disposition: the biological fate of chemicals 31 1461-73

Feenstra K A, Starikov E B, Urlacher V B, Commandeur J N and Vermeulen N P 2007 Combining substrate dynamics, binding statistics, and energy barriers to rationalize regioselective hydroxylation of octane and lauric acid by CYP102A1 and mutants Protein Sci 16 420-31

Feher V A, Baldwin E P and Dahlquist F W 1996 Access of ligands to cavities within the core of Nature structural biology 3

García A E 1992 Large-amplitude nonlinear motions in proteins Physical review letters 682696

Girvan H M, Dunford A J, Neeli R, Ekanem I S, Waltham T N, Joyce M G, Leys D, Curtis R A, Williams P, Fisher K, Voice M W and Munro A W 2011 Flavocytochrome P450 BM3 mutant W1046A is a NADH-dependent fatty acid hydroxylase: implications for the mechanism of electron transfer in the P450 BM3 dimer Archives of biochemistry and biophysics 507 75-85

Girvan H M, Marshall K R, Lawson R J, Leys D, Joyce M G, Clarkson J, Smith W E, Cheesman M R and Munro A W 2004 Flavocytochrome P450 BM3 mutant A264E undergoes substratedependent formation of a novel heme iron ligand set J Biol Chem 279 23274-86

Girvan H M, Waltham T N, Neeli R, Collins H F, McLean K J, Scrutton N S, Leys D and Munro A W 2006 Flavocytochrome P450 BM3 and the origin of CYP102 fusion species Biochem Soc Trans 34 1173-7

Guengerich F P 2001 Common and uncommon cytochrome P450 reactions related to metabolism and chemical toxicity Chem Res Toxicol 14 611-50

Haines D C, Tomchick D R, Machius M and Peterson J A 2001 Pivotal role of water in the mechanism of P450BM-3 Biochemistry 40 13456-65

Hazzard J T, Govindaraj S, Poulos T L and Tollin G 1997 Electron transfer between the FMN and heme domains of cytochrome P450BM-3. Effects of substrate and CO J Biol Chem $2727922-$ 6

Helms V, Deprez E, Gill E, Barret C, Hui Bon Hoa G and Wade R C 1996 Improved binding of cytochrome P450cam substrate analogues designed to fill extra space in the substrate binding pocket Biochemistry 35 1485-99

Huang W-C, Ellis J, Moody P C, Raven E L and Roberts G C 2013 Redox-linked domain movements in the catalytic cycle of cytochrome p450 reductase Structure 21 1581-9

Humphrey W, Dalke A and Schulten K 1996 VMD: visual molecular dynamics Journal of molecular graphics 14 33-8

Isralewitz B, Gao M and Schulten K 2001 Steered molecular dynamics and mechanical functions of proteins Current opinion in structural biology $11224-30$

Jones G, Willett P, Glen R C, Leach A R and Taylor R 1997 Development and validation of a genetic algorithm for flexible docking Journal of molecular biology $\mathbf{2 6 7}$ 727-48

Jovanovic T and McDermott A E 2005 Observation of ligand binding to cytochrome P450 BM-3 by means of solid-state NMR spectroscopy J Am Chem Soc 127 13816-21

Joyce M G, Ekanem I S, Roitel O, Dunford A J, Neeli R, Girvan H M, Baker G J, Curtis R A, Munro A W and Leys D 2012 The crystal structure of the FAD/NADPH-binding domain of flavocytochrome P450 BM3 The FEBS journal 279 1694-706

Kasim M, Chen H C and Swenson R P 2009 Functional characterization of the re-face loop spanning residues 536-541 and its interactions with the cofactor in the flavin mononucleotide-binding domain of flavocytochrome P450 from Bacillus megaterium Biochemistry 48 5131-41

Kuper J, Tee K L, Wilmanns M, Roccatano D, Schwaneberg U and Wong T S 2012a The role of active-site Phe87 in modulating the organic co-solvent tolerance of cytochrome P450 BM3 monooxygenase Acta Crystallogr F 68 1013-7

Kuper J, Wong T S, Roccatano D, Wilmanns M and Schwaneberg U 2007a Understanding a mechanism of organic cosolvent inactivation in heme monooxygenase P450BM-3 J Am Chem Soc 129 5786-+

Kuper J, Wong T S, Roccatano D, Wilmanns M and Schwaneberg U 2007b Understanding the mechanism of organic co-solvent inactivation in heme monooxygenase P450 BM-3 J. Am. Chem. Soc. 129 5786-7 
Kuper K, Tee K L, Wilmanns M, Roccatano D, Schwaneberg U and Wong T S 2012b The role of active-site Phe87 in modulating the organic co-solvent tolerance of cytochrome P450 BM3 monooxygenase Acta Cryst. Sect. F F68 1013-7

Lee E H, Hsin J, Sotomayor M, Comellas G and Schulten K 2009 Discovery Through the Computational Microscope Structure 17 1295-306

Ludemann S K, Lounnas V and Wade R C 2000a How do substrates enter and products exit the buried active site of cytochrome P450cam? 1. Random expulsion molecular dynamics investigation of ligand access channels and mechanisms Journal of molecular biology 303 797-811

Ludemann S K, Lounnas V and Wade R C 2000b How do substrates enter and products exit the buried active site of cytochrome P450cam? 2. Steered molecular dynamics and adiabatic mapping of substrate pathways Journal of molecular biology 303 813-30

MacKerell A, Wiórkiewicz-Kuczera J and Karplus M 1995 CHARMM22 Parameter Set Harvard University Department of Chemistry, Cambridge, $M A$

Marcus R A and Sutin N 1985 Electron transfers in chemistry and biology Biochim Biophys Acta 811 265-322

McCammon J A and Harvey S C 1987 Dynamics of proteins and nucleic acids (Cambridge Cambridgeshire ; New York: Cambridge University Press)

Meunier B, De Visser S P and Shaik S 2004 Mechanism of oxidation reactions catalyzed by cytochrome P450 enzymes Chemical reviews 104 3947-80

Moser C C, Keske J M, Warncke K, Farid R S and Dutton P L 1992 Nature of biological electron transfer Nature 355 796-802

Munro A W, Daff S, Coggins J R, Lindsay J G and Chapman S K 1996 Probing electron transfer in flavocytochrome P-450 BM3 and its component domains European journal of biochemistry / FEBS 239 403-9

Munro A W, Girvan H M and McLean K J 2007 Cytochrome P450--redox partner fusion enzymes Biochim Biophys Acta 1770 345-59

Munro A W, Leys D G, McLean K J, Marshall K R, Ost T W, Daff S, Miles C S, Chapman S K, Lysek D A, Moser C C, Page C C and Dutton P L 2002a P450 BM3: the very model of a modern flavocytochrome Trends Biochem Sci 27 250-7

Munro A W, Leys D G, McLean K J, Marshall K R, Ost T W B, Daff S, Miles C S, Chapman S K, Lysek D A, Moser C C, Page C C and Dutton P L 2002b P450BM3: the very model of a modern flavocytochrome Trends Biochem Sci 27 250-7

Munro A W, Lindsay J G, Coggins J R, Kelly S M and Price N C 1994 Structural and Enzymological Analysis of the Interaction of Isolated Domains of Cytochrome-P-450 Bm3 Febs Letters 343 70-4

Munro A W, Noble M A, Miles C S, Daff S N, Green A J, Quaroni L, Rivers S, Ost T W, Reid G A and Chapman S K 1999 Flavocytochrome P-450 BM3: a paradigm for the analysis of electron transfer and its control in the P-450s Biochem Soc Trans 27 190-6

Narhi L O and Fulco A J 1986 Characterization of a catalytically self-sufficient 119,000-dalton cytochrome P-450 monooxygenase induced by barbiturates in Bacillus megaterium $J$ Biol Chem 261 7160-9

Narhi L O and Fulco A J 1987a Identification and Characterization of 2 Functional Domains in Cytochrome-P-450bm-3, a Catalytically Self-Sufficient Monooxygenase Induced by Barbiturates in Bacillus-Megaterium J Biol Chem 262 6683-90

Narhi L O and Fulco A J 1987b Identification and characterization of two functional domains in cytochrome P-450BM-3, a catalytically self-sufficient monooxygenase induced by barbiturates in Bacillus megaterium J Biol Chem 262 6683-90

Nazor J, Dannenmann S, Adjei R O, Fordjour Y B, Ghampson I T, Blanusa M, Roccatano D and Schwaneberg U 2008 Laboratory evolution of P450 BM3 for mediated electron transfer yielding an activity-improved and reductase-independent variant Protein engineering, design \& selection: PEDS 21 29-35

Nazor J and Schwaneberg U 2006 Laboratory evolution of P450 BM-3 for mediated electron transfer Chembiochem : a European journal of chemical biology 7 638-44

Neeli R, Girvan H M, Lawrence A, Warren M J, Leys D, Scrutton N S and Munro A W 2005 The dimeric form of flavocytochrome P450 BM3 is catalytically functional as a fatty acid hydroxylase FEBS Lett 579 5582-8

Noble M A, Miles C S, Chapman S K, Lysek D A, MacKay A C, Reid G A, Hanzlik R P and Munro A W 1999 Roles of key active-site residues in flavocytochrome P450 BM3 The Biochemical journal 339 ( Pt 2) 371-9 
Oprea T I, Hummer G and García A E 1997 Identification of a functional water channel in cytochrome P450 enzymes Proceedings of the National Academy of Sciences 94 2133-8

Ost T W, Miles C S, Murdoch J, Cheung Y, Reid G A, Chapman S K and Munro A W 2000 Rational re-design of the substrate binding site of flavocytochrome P450 BM3 FEBS Lett 486 173-7

Page C C, Moser C C, Chen X and Dutton P L 1999 Natural engineering principles of electron tunnelling in biological oxidation-reduction Nature 402 47-52

Paulsen M D and Ornstein R L 1995 Dramatic differences in the motions of the mouth of open and closed cytochrome P450 BM-3 by molecular dynamics simulations Proteins: Stuct. Func. Bioinformatics 21 237-43

Petřek M, Otyepka M, Banáš P, Košinová P, Koča J and Damborský J 2006 CAVER: a new tool to explore routes from protein clefts, pockets and cavities BMC bioinformatics 7316

Pleiss J 2014 Systematic Analysis of Large Enzyme Families: Identification of Specificity-and Selectivity-Determining Hotspots ChemCatChem 6 944-50

Poulos T L 2003 Cytochrome P450 flexibility Proceedings of the National Academy of Sciences of the United States of America $10013121-2$

Pylypenko O and Schlichting I 2004 Structural aspects of ligand binding to and electron transfer in bacterial and fungal P450s Annu Rev Biochem 73 991-1018

Ravichandran K G, Boddupalli S S, Hasermann C A, Peterson J A and Deisenhofer J 1993 Crystal structure of hemoprotein domain of P450BM-3, a prototype for microsomal P450's Science $261731-6$

Roccatano D 2007 Nanostructured Soft Matter: Experiment, Theory, Simulation and Perspectives, ed A V Zvelindovsky: Springer )

Roccatano D 2008 Computer simulations study of biomolecules in non-aqueous or cosolvent/water mixture solutions Current Protein and Peptide Science 9 407-26

Roccatano D, Fioroni M, Zacharias M and Colombo G 2005a Effect of hexafluoroisopropanol alcohol on the structure of melittin: A molecular dynamics simulation study Protein Sci 14 2582-9

Roccatano D, Wong T S, Schwaneberg U and Zacharias M 2005b Structural and dynamic properties of cytochrome P450 BM-3 in pure water and in a dimethylsulfoxide/water mixture Biopolymers $78259-67$

Roccatano D, Wong T S, Schwaneberg U and Zacharias M 2006a Toward understanding the inactivation mechanism of monooxygenase P450 BM-3 by organic cosolvents: a molecular dynamics simulation study Biopolymers 83 467-76

Roccatano D, Wong T S, Schwaneberg U and Zacharias M 2006b Toward understanding the inactivation mechanism of monooxygenase P450 BM-3 by organic cosolvents: A molecular dynamics simulation study Biopolymers 83 467-76

Seifert A, Antonovici M, Hauer B and Pleiss J 2011 An efficient route to selective bio-oxidation catalysts: an iterative approach comprising modeling, diversification, and screening, based on CYP102A1 Chembiochem : a European journal of chemical biology 12 1346-51

Seifert A and Pleiss J 2012 Identification of selectivity determinants in CYP monooxygenases by modelling and systematic analysis of sequence and structure Current drug metabolism 13197 202

Seng Wong T, Arnold F H and Schwaneberg U 2004 Laboratory evolution of cytochrome P450 BM-3 monooxygenase for organic cosolvents Biotechnology and bioengineering 85 351-8

Sevrioukova I and Peterson J A 1996 Domain-domain interaction in cytochrome P450BM-3 Biochimie $78744-51$

Sevrioukova I, Shaffer C, Ballou D P and Peterson J A 1996a Equilibrium and transient state spectrophotometric studies of the mechanism of reduction of the flavoprotein domain of P450BM-3 Biochemistry 35 7058-68

Sevrioukova I, Truan G and Peterson J A 1996b The flavoprotein domain of P450BM-3: Expression, purification, and properties of the flavin adenine dinucleotide- and flavin mononucleotidebinding subdomains Biochemistry 35 7528-35

Sevrioukova I F, Li H, Zhang H, Peterson J A and Poulos T L 1999 Structure of a cytochrome P450redox partner electron-transfer complex Proceedings of the National Academy of Sciences of the United States of America 96 1863-8

Sligar S G, Makris T M and Denisov I G 2005 Thirty years of microbial P450 monooxygenase research: peroxo-heme intermediates--the central bus station in heme oxygenase catalysis Biochemical and biophysical research communications 338 346-54

Stierand K, Maaß P C and Rarey M 2006 Molecular complexes at a glance: automated generation of two-dimensional complex diagrams Bioinformatics 22 1710-6 
Stjernschantz E, van Vugt-Lussenburg B M, Bonifacio A, de Beer S B, van der Zwan G, Gooijer C, Commandeur J N, Vermeulen N P and Oostenbrink C 2008 Structural rationalization of novel drug metabolizing mutants of cytochrome P450 BM3 Proteins 71 336-52

Tee K L, Roccatano D, Stolte S, Arning J, Bernd J and Schwaneberg U 2008 Ionic liquid effects on the activity of monooxygenase P450BM-3 Green Chem 10 117-23

Udit A K, Hill M G, Bittner V G, Arnold F H and Gray H B 2004 Reduction of dioxygen catalyzed by pyrene-wired heme domain cytochrome P450 BM3 electrodes J Am Chem Soc 126 10218-9

van Gunsteren W F, Daura X and Mark A E 1998 GROMOS force field Encyclopedia of computational chemistry

van Rantwijk F, Madeira Lau R and Sheldon R A 2003 Biocatalytic transformations in ionic liquids Trends in Biotech. 21 131-8

Verma R, Schwaneberg U and Roccatano D 2013 Conformational Dynamics of the FMN-Binding Reductase Domain of Monooxygenase P450BM-3 J Chem Theory Comput 9 96-105

Verma R, Schwaneberg U and Roccatano D 2014 Insight into the Redox Partner Interaction Mechanism in Cytochrome P450BM-3 Using Molecular Dynamics Simulations Biopolymers 101 197-209

Wade R C, Winn P J, Schlichting I and Sudarko 2004 A survey of active site access channels in cytochromes P450 Journal of inorganic biochemistry 98 1175-82

Warman A J, Roitel O, Neeli R, Girvan H M, Seward H E, Murray S A, McLean K J, Joyce M G, Toogood H, Holt R A, Leys D, Scrutton N S and Munro A W 2005 Flavocytochrome P450 BM3: an update on structure and mechanism of a biotechnologically important enzyme Biochem Soc Trans 33 747-53

Whitehouse C J, Bell S G and Wong L L 2012 P450(BM3) (CYP102A1): connecting the dots Chem Soc Rev 41 1218-60

Winn P J, Ludemann S K, Gauges R, Lounnas V and Wade R C 2002 Comparison of the dynamics of substrate access channels in three cytochrome P450s reveals different opening mechanisms and a novel functional role for a buried arginine Proceedings of the National Academy of Sciences of the United States of America $995361-6$

Wittung-Stafshede P 2002 Role of cofactors in protein folding Acc Chem Res 35 201-8

Wong L L 1998 Cytochrome P450 monooxygenases Curr Opin Chem Biol 2 263-8

Wong T S, Arnold F H and Schwaneberg U 2004 Laboratory evolution of cytochrome p450 BM-3 monooxygenase for organic cosolvents Biotechnology and bioengineering 85 351-8

Wong T S, Wu N, Roccatano D, Zacharias M and Schwaneberg U 2005 Sensitive assay for laboratory evolution of hydroxylases toward aromatic and heterocyclic compounds Journal of biomolecular screening 10 246-52 